\title{
Pseudo Gallbladder sign in biliary atresia-an imaging pitfall
}

\author{
Seerat Aziz • Yvette Wild • Philip Rosenthal • \\ Ruth B. Goldstein
}

Received: 28 October 2010 /Revised: 31 December 2010 / Accepted: 5 January 2011 / Published online: 16 March 2011

(C) The Author(s) 2011. This article is published with open access at Springerlink.com

\begin{abstract}
Background Ultrasound (US) is used to identify causes of neonatal cholestasis. We describe a potential sonographic pitfall, the "pseudo gallbladder," in biliary atresia (BA).

Objective To describe the Pseudo Gallbladder sign (PsGB sign).

Materials and methods Sonograms/clinical records of 20 confirmed BA infants and 20 non-BA cases were reviewed retrospectively. For the BA group, preoperative sonography and surgical and pathological findings were examined. For the non-BA group, sonographic features and pathological findings were examined. The PsGB sign is defined as a fluid-filled structure, located in the expected region of the gallbladder, measuring $\leq 15 \mathrm{~mm}$ in length but without a well-defined or normal-appearing gallbladder wall.

Results A recognizable gallbladder and normal gallbladder wall were present in all non-BA infants. However, none of the BA infants had a sonographically normal gallbladder. Seventy-three percent of BA patients had a PsGB, and in $27 \%$ no gallbladder or gallbladder-like structure was detected.
\end{abstract}

\section{CME Activity}

This article has been selected as the CME activity for the current month. Please visit the SPR website at www.pedrad.org on the Education page and follow the instructions to complete this CME activity.

\section{S. Aziz $(\square) \cdot$ R. B. Goldstein}

Department of Radiology and Biomedical Imaging,

University of California, San Francisco,

505 Parnassus Ave., Box 0628, San Francisco,

CA 94143-0628, USA

e-mail: seerat.aziz@ucsf.edu

e-mail: seera.aziz@sbcglobal.net

Y. Wild $\cdot$ P. Rosenthal

Department of Pediatrics, University of California, San Francisco,

San Francisco, CA, USA
Conclusion A gallbladder-like structure in BA is common and can be misinterpreted as a normal gallbladder, delaying diagnosis and therapy. Recognition of this imaging pitfall, described here as the pseudo gallbladder sign, will help avoid this error.

Keywords Biliary atresia $\cdot$ Imaging pitfall $\cdot$ Pseudo gallbladder sign · Ultrasound

\section{Introduction}

Neonatal cholestasis, defined as conjugated hyperbilirubinemia occurring within the first 90 days of life, may be caused by biliary atresia (BA), infection, metabolic disorders such as alpha-1 antitrypsin deficiency (A1AT), neonatal hepatitis and choledochal cysts [1]. Of these, BA and idiopathic neonatal hepatitis comprise the majority of cases, with some authors describing BA as being the more common etiology $[1,2]$. In the United States, BA occurs in less than 1 per 10,000 live births [3, 4]. BA is characterized by a progressive obliteration of the intra- and extrahepatic biliary ducts and is treated by surgical portoenterostomy (Kasai procedure) and/or liver transplantation. Importantly, if untreated, BA can rapidly advance to biliary cirrhosis and death. Therefore it is essential to distinguish BA from other causes of cholestasis in the first 2 to 3 months of life.

Diagnostic methods utilized to distinguish BA from other causes of neonatal cholestatic jaundice include sonography, liver biopsy and intraoperative cholangiography. In the last two decades, a few sonographic observations have been described to aid in the diagnosis of BA [5-12] including the triangular cord sign (TC $>4 \mathrm{~mm}$ ), decreased gallbladder contractility, a small gallbladder length less than $15 \mathrm{~mm}$ described by Park et al. [6] or less than $19 \mathrm{~mm}$ described by 
Tan Kendrick et al. [11]. The latter authors previously described the "gallbladder ghost triad" (atretic gallbladder length $<19 \mathrm{~mm}$, thin or absent mucosal lining or indistinct wall, and an irregular gallbladder contour) [11].

In some children with confirmed BA, we have observed a fluid-filled structure in the mid-liver, without a well-defined wall, that might be mistaken for a normal gallbladder. While our finding is comparable to the ghost triad reported by Tan Kendrick et al. [11], we have termed this structure the Pseudo Gallbladder sign (PsGB sign) as a reminder that this structure should not be confused for a normal gallbladder, potentially delaying accurate diagnosis. We describe the PsGB sign and its prevalence among infants with proven BA to increase awareness of this potential pitfall in the sonography of infants with cholestasis.

\section{Materials and methods}

A retrospective review of clinical, pathological and imaging records of all infants with evidence of a direct hyperbilirubinemia and cholestasis from 2004 to 2009 was performed. Infants with cholestasis related to TPN or sepsis were excluded from the study. The remaining included 20 infants with known BA and 20 infants with neonatal cholestasis from non-BA causes as listed below. The UCSF Committee on Human Research (IRB) approved the study. The committee waived consent because some of the children are deceased and some are no longer followed at UCSF. All infants were less than 5 months of age at their initial US evaluation at UCSF.

Inclusion criteria for the study were preoperative sonograms at our center and definitive diagnosis of BA proven by liver biopsy, intra-operative cholangiogram and surgical pathology. Exclusion criteria included Kasai portoenterostomy or liver transplant prior to initial sonogram and lack of preoperative sonographic imaging at UCSF. Of the 20 BA infants, five were excluded because they had a Kasai procedure prior to their first US exam. The remaining 15 BA cases were included in this study. Histopathological confirmation of BA from the surgical specimens was used as the diagnostic gold standard.

Although in the past, preoperative nuclear imaging has been performed at our institution to assess patency of the biliary tree, we have abandoned this approach as both biliary atresia and severe intrahepatic cholestasis can demonstrate lack of isotope excretion into the intestine. Therefore, a HIDA scan was not performed in any of the infants included in this study.

The 20 infants with neonatal cholestasis with causes other than BA presenting during the same time frame were used as controls, called the non-BA group. A retrospective review of case and imaging records of these infants identified the underlying etiology in these cases, which included Alagille syndrome, alpha-1 antitrypsin deficiency, neonatal sclerosing cholangitis, neonatal hepatitis, cryptogenic cirrhosis, giant cell hepatitis and cholestasis of unknown etiology. Although the control infants were not age-matched by exact day of life to those in the study group, the average age in these groups was 2.15 and 2.13 months, respectively. All infants were NPO at a minimum of $4 \mathrm{~h}$ at the time of the sonographic evaluation.

US studies were retrieved and viewed from PACS (picture archiving and communication systems). These sonograms were performed using Sequoia US machines (Siemens Medical Solutions, Erlangen, Germany) using multifocused vector and linear transducers ranging from 4 to $8 \mathrm{MHz}$. Sonographic images of both groups (BA and non-BA infants) were reviewed by two experienced sonologists (RBG and SA) who have 31 years of combined post-residency experience in pediatric US. Images were assessed concurrently by both reviewers with consensus reached at the time of the review. Images were evaluated for the presence or absence of a gallbladder or a gallbladder-like structure including its twodimensional measurements, for the presence of a gallbladder wall and the triangular cord sign.

For the triangular cord sign (TC), tissue thickness anterior to the right portal vein greater than $4 \mathrm{~mm}$ qualified as abnormal, based on Lee et al.'s [7] measurement criterion. A normal gallbladder was defined as $>15 \mathrm{~mm}$ length and displaying a normal-appearing wall. A normal gallbladder wall was defined as a measurable interface composed of at least one peripheral hypoechoic fibromuscular layer and one central echogenic mucosal interface with a relatively even thickness (Fig. 1). The PsGB sign was defined as a fluid-filled gallbladder-like structure located in the region of the main lobar fissure and measuring less than or equal to $15 \mathrm{~mm}$ in length, with a surrounding smooth or irregular bright margin but without a well-defined or normal-appearing gallbladder wall (Fig. 2). Surgical pathology findings were available for all infants.

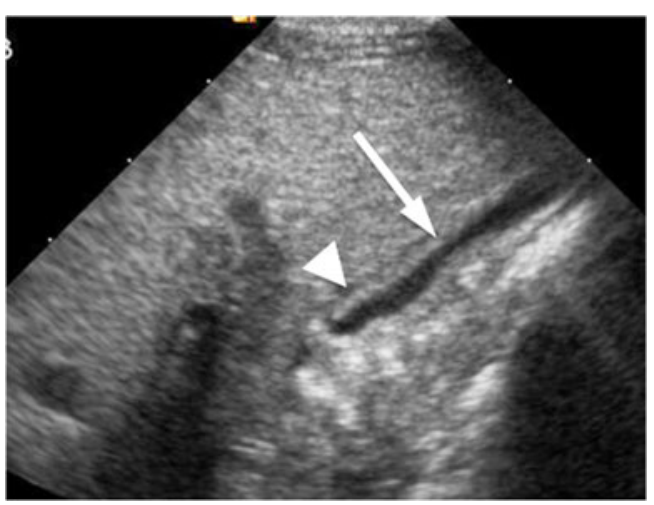

Fig. 1 Normal gallbladder wall. Normal sonographic appearance of the gallbladder in a 2-day-old neonate with no known hepatobiliary disease demonstrates a well-defined measurable normal gallbladder wall. Note the central echogenic mucosal interface (arrow) and peripheral thin fibromuscular layer (arrowhead) 
Fig. 2 The pseudo gallbladder sign (PsGB sign). Different appearances (arrows) of the PsGB sign, which is defined as a fluid-filled structure $<15 \mathrm{~mm}$ in length in the region of the interlobar fissure without a normal gallbladder wall (see Fig. 1 for a normal-appearing wall). a Notice the smooth contour of this PsGB compared with another PsGB (b) with an irregular margin. c Irregular contour in another PsGB. d PsGB appears as a very small, focal, nonspecific fluid collection. Note that a normal gallbladder wall is not seen in any of these cases
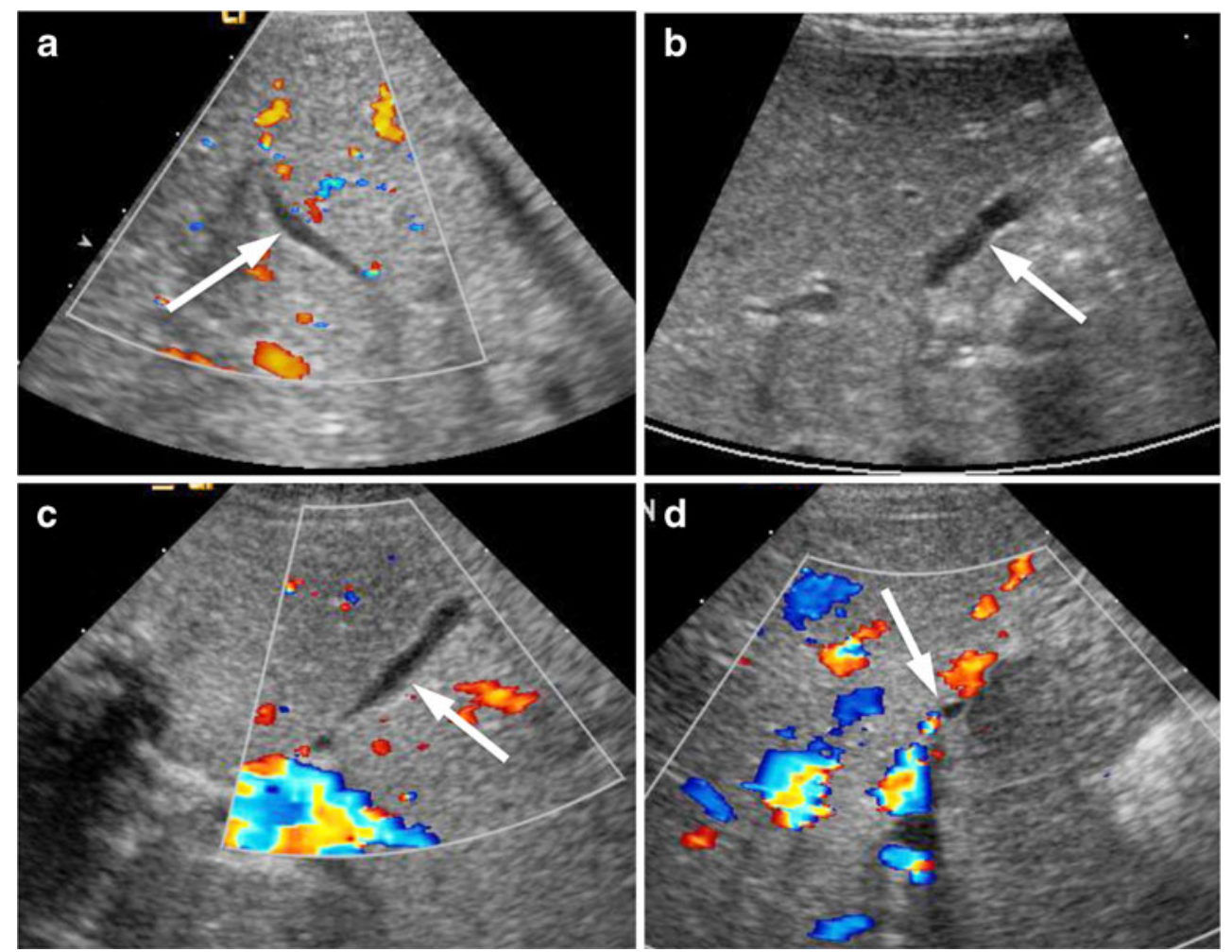

\section{Results}

Mean age at initial presentation was 2.1 months (range 0.14.6 months) for the BA group and 2.2 months (range 15 months) for the non-BA group. Of the 15 infants with pathologically proven BA (Tables 1 and 2), no gallbladder or gallbladder-like structure was detected on the abdominal sonogram or on pathological examination of the surgical tissue in four patients $(27 \%)$. In the remaining $11 \mathrm{BA}$ cases (73\%), a normal gallbladder was not seen. Instead, the sonogram showed a small gallbladder-like structure, indicated by the presence of a tubular fluid-filled shape in the
Table 1 Sonographic findings in both groups of infants
Biliary atresia infants $n=15$

Non-biliary atresia (Controls) $n=20$

\begin{tabular}{lll}
\hline $\begin{array}{l}\text { Normal GB } \\
\text { Yes }\end{array}$ & 0 & 20 \\
No & 15 & 0 \\
Pseudo gallbladder sign & & \\
Yes & 11 & 0 \\
No & & 20 \\
No gallbladder seen & 4 & 0 \\
PsGB/gallbladder length & & \\
$\leq 15$ mm & $9 / 11$ & 5 (Normal wall-Yes) \\
$>15$ mm & $2 / 11$ (Normal wall-No) & 15 \\
Ave length & $11.4 \mathrm{~mm}$ & $20.9 \mathrm{~mm}$ \\
Ave width & $3.2 \mathrm{~mm}$ & $5.8 \mathrm{~mm}$ \\
Normal gallbladder wall & & 19 \\
Yes & $2 / 11$ & 1 \\
No & $9 / 11$ & 1 \\
Triangular cord sign & & 19 \\
Yes & $9 / 15$ & $2.4 \mathrm{~mm}$ \\
No & $6 / 15$ & \\
Ave width & $5.9 \mathrm{~mm}$ & \\
\hline
\end{tabular}


Table 2 Age at Kasai for the biliary atresia cases, sonographic features and pathological findings
$P s G B$ Pseudo gallbladder sign, US Ultrasound, GB Gallbladder, Hypopl Hypoplastic

\begin{tabular}{lllllll}
\hline Case \# & $\begin{array}{l}\text { Age at US } \\
\text { (in days) }\end{array}$ & $\begin{array}{l}\text { Days from US } \\
\text { to Kasai }\end{array}$ & $\begin{array}{l}\text { Age at Kasai } \\
\text { (in days) }\end{array}$ & PsGB on US & GB wall & $\begin{array}{l}\text { Surgical } \\
\text { pathology }\end{array}$ \\
\hline 1 & 61 & 2 & 63 & $6 \times 2 \mathrm{~mm}$ & No & No GB \\
2 & 45 & 84 & 129 & $7 \times 5 \mathrm{~mm}$ & Yes & No GB \\
3 & 43 & 9 & 52 & No & No & No GB \\
4 & 67 & 1 & 68 & $3 \times 2 \mathrm{~mm}$ & No & No GB \\
5 & 139 & 3 & 142 & $8 \times 3 \mathrm{~mm}$ & Yes & Not true GB \\
6 & 112 & 1 & 113 & $12 \times 3 \mathrm{~mm}$ & No & Hypopl GB \\
7 & 69 & 3 & 72 & No & No & No GB \\
8 & 10 & 48 & 58 & No & No & No GB \\
9 & 4 & 47 & 51 & $21 \times 4 \mathrm{~mm}$ & No & No GB \\
10 & 89 & 3 & 92 & $11 \times 3 \mathrm{~mm}$ & No & No GB \\
11 & 3 & 49 & 52 & No & No & No GB \\
12 & 45 & 2 & 47 & $17 \times 2$ & No & Atretic GB \\
13 & 65 & 4 & 69 & $15 \times 3$ & No & Hypopl GB \\
14 & 65 & 5 & 70 & $15 \times 3$ & No & Hypopl GB \\
15 & 31 & 44 & 75 & $10 \times 5$ & No & No GB epith \\
\hline
\end{tabular}

expected region of the gallbladder in the interlobar fissure, with an average length of $11 \mathrm{~mm}$ and an average width of $3 \mathrm{~mm}$. These findings constituted the Pseudo Gallbladder sign (PsGB sign).

On subsequent histopathological examination of surgical liver tissue of the 11 PsGB cases, no gallbladder was identified in 5, 4 had hypoplastic or atretic gallbladders and 2 had tissue that was not consistent with a true gallbladder/no epithelium (Table 2). Sonographically, all the pseudo gallbladders were smaller than or equal to the discriminatory length of $15 \mathrm{~mm}$ except for two (17 $\mathrm{mm}$ and $21 \mathrm{~mm}$ length). However, the two longer PsGBs were not normal-appearing on US exam, had irregular wavy contours and did not have a normal wall (Fig. 3). The 17-mm structure correlated with an atretic gallbladder on pathological evaluation, while no gallbladder was seen on pathology for the 21-mm case. Hence, a normal gallbladder was not seen by US in any infant with proven BA.

Moreover, a normal gallbladder wall was not identified in 9/11 (82\%) cases demonstrating the PsGB sign. Of the two PsGB patients (18\%) who did have a normal-appearing wall, the sonographically measured lengths were very small at $8 \mathrm{~mm}$ and $7 \mathrm{~mm}$, with the first shown to be an atretic gallbladder at pathology, while no gallbladder tissue was seen in the other.

In the control group (Table 1) a normal-size gallbladder was demonstrated in 15/20 (75\%) non-BA cases. Of these, there was one case where a normal echogenic wall was not clearly observed but the gallbladder measured $19 \times 10 \mathrm{~mm}$, much greater than the $15-\mathrm{mm}$ cutoff limit established by Park et al. [6]. This was therefore interpreted as a normal gallbladder. The remaining five (25\%) non-BA patients had a gallbladder length less than $15 \mathrm{~mm}$, but a normal echogenic gallbladder wall and shape was present in all five cases. Thus, a PsGB sign was not seen in any of the non-BA controls.

Using the 15-mm length cutoff described above, the difference in mean PsGB/gallbladder length was statistically
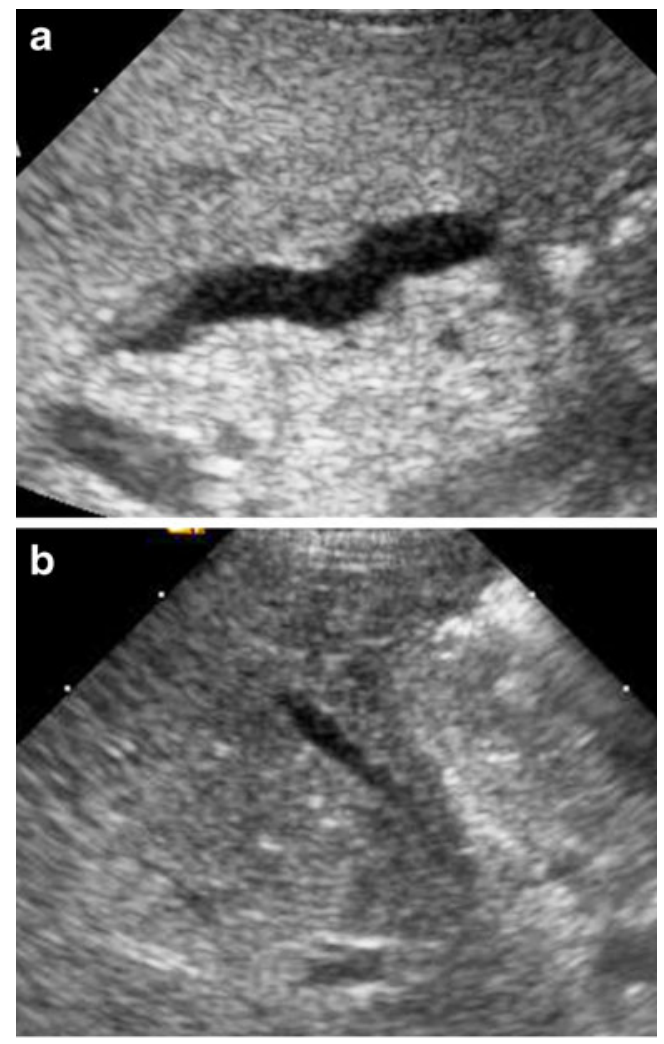

Fig. 3 Two cases of PsGB with length $>15 \mathrm{~mm}$ but with absence of a definable wall. a PsGB has a length of $21 \mathrm{~mm}$ but with wavy contour and no definable wall. b PsGB has a length of $17 \mathrm{~mm}$ but with irregular margins and no measurable wall 
significant $(p<0.05)$ between the BA group (mean length $11.4 \mathrm{~mm} \pm 5.3, n=15$ ) and the non-BA group (mean length $21 \mathrm{~mm} \pm 10, n=20$ ) (Table 3). The triangular cord sign was identified in $9 / 15(60 \%)$ BA infants and in only $1 / 20(5 \%)$ non-BA infants (Table 1). In the latter case, the TC measurement was $4.3 \mathrm{~mm}$, only minimally greater than the 4-mm cutoff. The average TC width was greater in the BA group than in the non-BA controls $(5.9 \mathrm{~mm}$ vs. $2.4 \mathrm{~mm}$, $p<0.05$ ) (Table 3).

\section{Discussion}

Neonatal cholestasis has various causes with different treatment protocols. It is important to differentiate between BA and other causes of infantile cholestasis because BA is treated surgically (with some degree of urgency) while other causes might be managed medically. Initial treatment of BA is the Kasai portoenterostomy, which can re-establish bile flow in up to $80 \%$ of cases if performed prior to 60 days of age $[1,13]$. This number decreases to only $20 \%$ if the operation is performed after 90 days of life-hence the importance of early diagnosis. A liver transplant is required if the Kasai procedure is unsuccessful. To date, BA remains the leading indication for liver transplantation in children in the United States [14].

BA is characterized by the obliteration of all or part of the extrahepatic bile ducts and/or the most central part of the intrahepatic bile ducts. The precise etiology of BA has not been established, although several pathogenetic mechanisms have been proposed including a developmental disruption of the biliary tree occurring antenatally (fetal form in approximately $20 \%$ of cases of BA) and an immunological response to a viral pathogen (perinatal form $70-80 \%$ of cases) $[15,16]$. Current thinking suggests an association of an autoimmune response to a viral infection such as cytomegalovirus, group $\mathrm{C}$ rotavirus or reovirus type 3 [16-20]. Others have described the additional possible role of human papilloma virus [21] and retroviruses [22] as well as several other risk factors including advanced maternal age, increased parity, small for gestational age, low birth weight and prematurity [18].

Sonography is the initial imaging modality of choice when evaluating the neonate with cholestasis and helps to

Table 3 Statistical significance of various parameters between BA and non-BA groups using Student $t$-test

\begin{tabular}{lll}
\hline & $P$ value & Statistically significant at $P<0.05$ \\
\hline PsGB/GB length & 0.008 & Yes \\
PsGB/GB width & 0.098 & No \\
TC sign & $2.43 \mathrm{E}-10$ & Yes \\
Age of patient & 0.95 & No \\
\hline
\end{tabular}

exclude overt anatomical causes of obstruction such as a choledochal cyst. Initial US research in BA focused on the presence or absence of the gallbladder and whether the gallbladder contracted following feeding [23-25]. The presence of a normal gallbladder (without other abnormal findings in the biliary tree) is usually thought to essentially exclude the diagnosis of BA. In this study we describe a potential important imaging pitfall of a pseudo gallbladder sign, which if misinterpreted as a normal gallbladder could delay important diagnostic testing and the correct diagnosis.

Since BA can occur at any level of the biliary tree, the presence of a gallbladder, cystic duct and a distal bile duct does not completely exclude a more proximal ductal atresia [9]. Clinical and histopathological suspicion of BA can be confirmed by an intraoperative cholangiogram [1], which shows failure of contrast agent reflux into the proximal/ intrahepatic ducts.

Attention has also been directed to the identification and dilatation of bile ducts, echotexture of the liver and the TC sign. Choi and co-workers [5] first described the TC sign in 1996 in BA patients as a tubular or triangular echogenic tissue (fibrous ductal remnant) adjacent to the portal vein and correlated this sonographic finding with a biopsyproved diagnosis of BA (Fig. 4). However, they recommended close follow-up of all patients given the possibility of false-positive and false-negative findings. Lee et al. [7] found that the TC sign has a 94\% PPV and NPV, 98\% specificity and $80 \%$ sensitivity. Compared to $73 \%$ sensitivity and $100 \%$ specificity for the TC sign, demonstrated by Humphrey and Stringer [26], our study shows $60 \%$ sensitivity and $95 \%$ specificity (Table 4). Li et al. [27] suggest that the TC sign should not be viewed as the only imaging criterion for diagnosing BA. Tan Kendrick et al. [11] have reported high sensitivity and specificity for the diagnosis of BA using the gallbladder ghost triad.

In our study, the reviewers were not blinded to the diagnosis at the time of the review and although there is an inherent bias in this method, the case and control groups were so clearly segregated based on the findings that we do not believe this would be significant to the general results. Of the 15 infants with pathologically proven BA, we found that no gallbladder was seen in $27 \%$ of our BA cases $(n=4)$, but that $73 \%(n=11)$ of our BA patients had a gallbladderlike structure - the pseudo gallbladder sign. This structure was $\leq 15 \mathrm{~mm}$ in length in the majority (9/11) of cases and is indicated by the presence of a small ovoid to tubular fluidfilled structure in the expected region of the gallbladder (Fig. 2). It is smaller than a normal gallbladder and can have irregular margins but without a well-defined or normal-appearing wall. The term "pseudo gallbladder" emphasizes the potential to misconstrue this finding as a normal gallbladder, which can distract from the diagnosis of biliary atresia. 


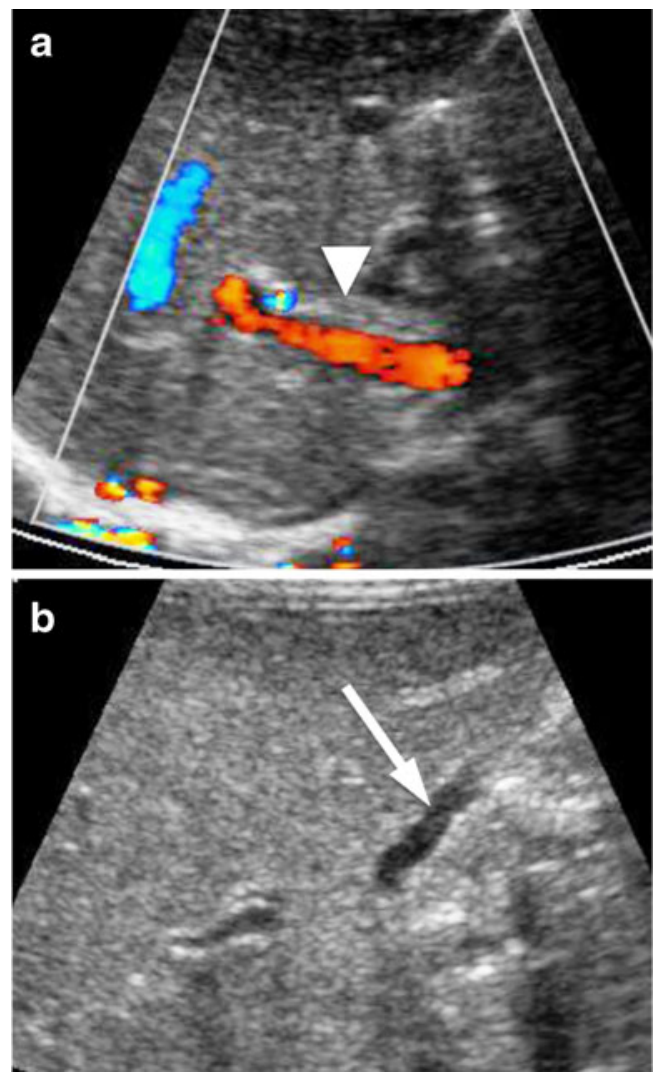

Fig. 4 The triangular cord sign (TC sign) in a BA patient. a The TC sign (arrowhead) measured $4.8 \mathrm{~mm}$ in this case of BA. b The PsGB sign in this case (arrow) is seen as a fluid-filled structure demonstrating an irregular contour but without a normal gallbladder wall

The presence or absence of a gallbladder wall helps distinguish a normal gallbladder from a PsGB[11]. A normal wall is measurable with a central echogenic mucosal interface and a thinner peripheral fibromuscular layer (Fig. 1), whereas the PsGB does not display a normal wall but rather has only a thin nonmeasurable echogenic rim around the fluid collection (Fig. 2). A normal gallbladder wall was absent in $82 \%$ of our BA cases with PsGB but was clearly identified in $95 \%$ of the non-BA control group. Absence of the gallbladder wall had an $87 \%$ sensitivity and 95\% specificity with a 93\% PPV for BA (Table 4).

Among the 11/15 BA cases demonstrating the PsGB sign, surgical pathology showed no gallbladder in five cases and a hypoplastic gallbladder in three, and the remaining three were labeled as "not a true gallbladder, atretic gallbladder, and no epithelium seen," respectively. Thus, there was no single pathological correlate to the PsGB in our study. Histologically, hyaline cartilage at the portal plate $[28,29]$, in the bile ducts [30] and in an atretic gallbladder [31], and squamous epithelium within biliary ductules [29] has been reported. However, the wide spectrum of previously described histopathological findings in BA is mostly without sonographic correlation. The complete absence of smooth muscle bundles in the wall of an atretic gallbladder, noted by Altamirano [31], might correspond to our observation of the absence of a sonographically normal gallbladder wall in most of our PsGB cases. The varied surgical pathology of the PsGB in our study and in other reported BA cases suggests the need for a larger clinical-radiological-pathological study in the future to provide a more definitive pathological correlate to the PsGB.

A common misconception is that the identification of what appears to be a gallbladder more or less dismisses the possibility of BA. We contend that awareness should be raised that a gallbladder-like structure can be present in association with $\mathrm{BA}$ and that its presence should not dissuade one from a diagnosis of BA.

\section{Conclusion}

The presence of a gallbladder-like structure, the Pseudo Gallbladder sign (PsGB sign), is a potential sonographic finding in infants with biliary atresia and should not lead to the erroneous assumption that a normal gallbladder is being identified. Awareness of this imaging pitfall will help prevent the inappropriate sonographic diagnostic exclusion of biliary atresia.

Moreover, a normal-appearing gallbladder wall is absent in a large proportion of biliary atresia cases $(82 \%)$ demonstrating the pseudo gallbladder sign. Therefore, in suspected cases of pseudo gallbladder, the associated lack of a definite gallbladder wall should increase the level of confidence in suggesting the diagnosis of biliary atresia.
Table 4 Sensitivity, specificity, positive predictive value (PPV) and negative predictive value (NPV) for various imaging criteria

\begin{tabular}{llll}
\hline & $\begin{array}{l}\text { TC sign in biliary atresia width } \\
\text { greater than } 4 \mathrm{~mm}\end{array}$ & $\begin{array}{l}\text { Absence of GB wall } \\
\text { indicating biliary atresia }\end{array}$ & $\begin{array}{l}\text { Presence of GB wall } \\
\text { indicating normal GB }\end{array}$ \\
\hline Sensitivity & $60 \%$ & $87 \%$ & $95 \%$ \\
Specificity & $95 \%$ & $95 \%$ & $82 \%$ \\
PPV & $90 \%$ & $93 \%$ & $90 \%$ \\
NPV & $76 \%$ & $90 \%$ & $90 \%$ \\
\hline
\end{tabular}


Open Access This article is distributed under the terms of the Creative Commons Attribution Noncommercial License which permits any noncommercial use, distribution, and reproduction in any medium, provided the original author(s) and source are credited.

\section{References}

1. Suchy FJ (2004) Neonatal cholestasis. Pediatr Rev 25:388-396

2. Balistreri WF (1985) Neonatal cholestasis. J Pediatr 106:171-184

3. Yoon PW, Bresee JS, Olney RS et al (1997) Epidemiology of biliary atresia: a population-based study. Pediatrics 99:376-382

4. Caton AR, Druschel CM, McNutt LA (2004) The epidemiology of extrahepatic biliary atresia in New York State, 1983-98. Paediatr Perinat Epidemiol 18:97-105

5. Choi SO, Park WH, Lee HJ et al (1996) 'Triangular cord': a sonographic finding applicable in the diagnosis of biliary atresia. $\mathrm{J}$ Pediatr Surg 31:363-366

6. Park WH, Choi SO, Lee HJ (1999) The ultrasonographic 'triangular cord' coupled with gallbladder images in the diagnostic prediction of biliary atresia from infantile intrahepatic cholestasis. J Pediatr Surg 34:1706-1710

7. Lee HJ, Lee SM, Park WH et al (2003) Objective criteria of triangular cord sign in biliary atresia on US scans. Radiology 229:395-400

8. Takamizawa S, Zaima A, Muraji T et al (2007) Can biliary atresia be diagnosed by ultrasonography alone? J Pediatr Surg 42:2093-2096

9. Ikeda S, Sera Y, Ohshiro H et al (1998) Gallbladder contraction in biliary atresia: a pitfall of ultrasound diagnosis. Pediatr Radiol 28:451-453

10. Farrant P, Meire HB, Mieli-Vergani G (2001) Improved diagnosis of extraheptic biliary atresia by high frequency ultrasound of the gall bladder. Br J Radiol 74:952-954

11. Tan Kendrick AP, Phua KB, Ooi BC et al (2003) Biliary atresia: making the diagnosis by the gallbladder ghost triad. Pediatr Radiol 33:311-315

12. Kanegawa K, Akasaka Y, Kitamura E et al (2003) Sonographic diagnosis of biliary atresia in pediatric patients using the 'triangular cord' sign versus gallbladder length and contraction. AJR 181:1387-1390

13. Kasai M (1983) Advances in treatment of biliary atresia. Jpn J Surg 13:265-276

14. Balistreri WF, Grand R, Hoofnagle JH et al (1996) Biliary atresia: current concepts and research directions. Summary of a symposium. Hepatology 23:1682-1692

15. Sokol RJ, Mack C (2001) Etiopathogenesis of biliary atresia. Semin Liver Dis 21:517-524
16. Mack CL (2007) The pathogenesis of biliary atresia: evidence for a virus-induced autoimmune disease. Semin Liver Dis 27:233242

17. Fischler B, Ehrnst A, Forsgren $M$ et al (1998) The viral association of neonatal cholestasis in Sweden: a possible link between cytomegalovirus infection and extrahepatic biliary atresia. J Pediatr Gastroenterol Nutr 27:57-64

18. Fischler B, Haglund B, Hjern A (2002) A population-based study on the incidence and possible pre- and perinatal etiologic risk factors of biliary atresia. J Pediatr 141:217222

19. Fischler B, Svensson JF, Nemeth A (2009) Early cytomegalovirus infection and the long-term outcome of biliary atresia. Acta Paediatr 98:1600-1602

20. Wilson GA, Morrison LA, Fields BN (1994) Association of the reovirus $\mathrm{S} 1$ gene with serotype 3 -induced biliary atresia in mice. $\mathrm{J}$ Virol 68:6458-6465

21. Drut R, Drut RM, Gomez MA et al (1998) Presence of human papillomavirus in extrahepatic biliary atresia. J Pediatr Gastroenterol Nutr 27:530-535

22. Mason AL, Xu L, Guo L et al (1998) Detection of retroviral antibodies in primary biliary cirrhosis and other idiopathic biliary disorders. Lancet 351:1620-1624

23. Green D, Carroll BA (1986) Ultrasonography in the jaundiced infant: a new approach. J Ultrasound Med 5:323-329

24. Ikeda S, Sera Y, Akagi M (1989) Serial ultrasonic examination to differentiate biliary atresia from neonatal hepatitis-special reference to changes in size of the gallbladder. Eur J Pediatr $148: 396-400$

25. Weinberger E, Blumhagen JD, Odell JM (1987) Gallbladder contraction in biliary atresia. AJR 149:401-402

26. Humphrey TM, Stringer MD (2007) Biliary atresia: US diagnosis. Radiology 244:845-851

27. Li SX, Zhang Y, Sun M et al (2008) Ultrasonic diagnosis of biliary atresia: a retrospective analysis of 20 patients. World J Gastroenterol 14:3579-3582

28. Mirkin LD, Knisely AS (1997) Hyaline cartilage at porta hepatis in extrahepatic biliary atresia. Pediatr Pathol Lab Med 17:587-591

29. Stahlschmidt J, Stringer MD, Wyatt J et al (2008) Histologic oddities at the porta hepatis in biliary atresia. J Pediatr Surg 43:1328-1332

30. Kashiwagi Y, Kawashima H, Ioi H et al (2007) Biliary atresia with cartilage formation. Pediatr Int 49:683-685

31. Altamirano E, Drut R (2008) Extrahepatic biliary atresia with cartilaginous metaplasia in the gallbladder remnants. J Clin Pathol 61:965-966 